\title{
PERCEPÇÕES DE PROFESSORES DA ÁREA DE CIÊNCIAS DA NATUREZA E MATEMÁTICA SOBRE A INTERDISCIPLINARIDADE
}

\author{
PERCEPTIONS OF TEACHERS OF THE AREA OF NATURE AND \\ MATHEMATICS SCIENCE ON INTERDISCIPLINARITY
}

\author{
Idmaura Calderaro Martins Galvão ${ }^{1}$ \\ Ana Carolina Biscalquini Talamoni ${ }^{2}$ \\ Isabel Cristina de Castro Monteiro ${ }^{3}$
}

\begin{abstract}
Resumo: Esta pesquisa tem por objetivo levantar e analisar as percepções de professores da área de Ciências da Natureza e Matemática acerca do tema Interdisciplinaridade aplicada ao Ensino de Ciências. Os professores participantes dessa pesquisa atuam em uma escola pública do interior do Estado de São Paulo, nas disciplinas de Ciências Biológicas, Matemática, Física, Química e Biologia. Aplicamos um questionário aberto aos professores, a fim de levantar suas ideias e analisar seus argumentos. Por meio dos referenciais de interdisciplinaridade propostos por Fourez (1994), Lenoir (2001) e Fazenda (2002) investigamos as argumentações desses professores com relação à inserção de atividades interdisciplinares na prática educativa, tomando por base o padrão de Toulmin (2006). Os resultados mostram indícios de que o trabalho interdisciplinar é defendido pelos docentes com argumentações lógicas e coerentes, indicando seu caráter dialógico, transformador e coerente com os objetivos que se pretende atingir para a formação dos alunos, no Ensino Médio. Os resultados indicam que o tema é relevante para a área de Ciências da Natureza e Matemática e pode contribuir para os estudos que tratam da emergência do tema na formação de professores.
\end{abstract}

Palavras-chave: Interdisciplinaridade; Ensino de Ciência; Argumentação.

\begin{abstract}
This research aims to raise and analyze the perceptions of teachers in the area of Natural Sciences and Mathematics about the theme Interdisciplinarity applied to Science Teaching. The teachers participating in this research work in a public school in the interior of the state of São Paulo, in the subjects of Biological Sciences, Mathematics, Physics, Chemistry and Biology. We applied an open questionnaire to teachers to raise their ideas and analyze their arguments. Through the references of interdisciplinarity proposed by Fourez (1994), Lenoir (2001) and Fazenda (2002) we investigated the arguments of these teachers regarding the insertion of interdisciplinary activities in educational practice, based on the pattern of Toulmin (2006). The results show evidence that the interdisciplinary work is defended by the teachers with logical and coherent arguments, indicating its dialogical, transformative and coherent character with the objectives that are intended to achieve the formation of students in high school. The results indicate that the theme is relevant to the area of Natural Sciences and Mathematics and may contribute to studies that address the emergence of the theme in teacher education.
\end{abstract}

Keywords: Interdisciplinarity; Science teaching; Argumentation.

\footnotetext{
${ }^{1}$ Doutoranda em Educação para a Ciência pela Universidade Estadual Paulista (UNESP). Universidade Estadual Paulista (UNESP), Bauru, São Paulo, Brasil. E-mail: idmaura@ gmail.com

${ }^{2}$ Doutora em Educação para a Ciência pela Universidade Estadual Paulista (UNESP). Professora Assistente Doutora do Instituto de Biociências do Campus do Litoral Paulista da Universidade Estadual Paulista (UNESP), São Vicente, São Paulo, Brasil. E-mail: ana.talamoni@unesp.br

${ }^{3}$ Doutora em Educação para a Ciência pela Universidade Estadual Paulista (UNESP). Professora Assistente Doutora do Departamento de Física e Química da Faculdade de Engenharia de Guaratinguetá da Universidade Estadual Paulista (UNESP), Guaratinguetá, São Paulo, Brasil. E-mail: monteiro@feg.unesp.br
} 


\section{Introdução}

A existência de duas culturas no delineamento e nas práticas científicas já é conhecida pela filosofia da ciência desde o ensaio de Snow (1995). Este dualismo reflete a construção histórica das ciências e pauta-se, sobretudo, na proposição da metodologia e da linguagem científicas que, a seu tempo, restringiram-se a fenômenos físicos e à linguagem matemática. Estes elementos foram fundamentais para o estabelecimento de uma ciência neutra e objetiva ao longo do século XIX, e para o desenvolvimento de programas de pesquisa circunscritos nas esferas disciplinares da ciência.

A organização da própria ciência e seu desenvolvimento culminou assim, naquilo que se entende hoje, por disciplinas científicas com campos de atuação bastante delimitados e reflete-se na estrutura do próprio conhecimento, essencialmente disciplinar, sobretudo durante a formação inicial dos futuros profissionais.

A disciplinarização do conhecimento passou assim, de uma forma de organização do mesmo a uma espécie de epistemologia. E esta epistemologia tem sido duramente criticada, no âmbito da filosofia da ciência, a partir da década de 1960 do século passado. O paulatino aprofundamento das questões e objetos de pesquisa em detrimento dos impactos sociais e da contextualização do mesmo, tem impedido que comunidades científicas avaliem apropriadamente o impacto de suas práticas e de suas descobertas. Além disto, por sua extrema especialização, tem se mantido alijada da sociedade e da cultura que as produz, impedindo que os conhecimentos gerados possam ser aplicados na compreensão mais abrangente da natureza e dos fenômenos socioambientais.

No contexto da modernidade tardia, no qual impõe-se problemas de natureza complexa e interdisciplinar como os relativos à bioética e ao meio ambiente, tentativas de se criar uma nova epistemologia, interdisciplinar, mostrou-se necessária e evidente.

\section{Referencial teórico}

O termo interdisciplinaridade foi encontrado pela primeira vez em 1937 no Journal of Education Sociology e o primeiro levantamento a respeito do desenvolvimento de atividades interdisciplinares ocorreu na década de 1960, por iniciativa da OECD (Centro de Pesquisa e Inovação Educacional da Organização para a Cooperação e o Desenvolvimento Econômico). Este mapeamento visava a reflexão e proposição de novas iniciativas com foco no desenvolvimento da ciência e no maior estreitamento dos laços e diálogos entre as universidades e a sociedade em geral. Em 1982 a mesma organização 
DOI: http://dx.doi.org/10.33238/ReBECEM.2019.v.3.n.3.23547

declarou prioridade de ações de cunho social para as universidades, que deveriam comprometer-se com os problemas sociais mais amplos e a resolução de problemas práticos do cotidiano (SOMMERMAN, 2005, p. 6-7).

A partir de então, diversos seminários de cunho internacional foram realizados com o objetivo de melhor debater e definir o movimento interdisciplinar, destacando-se assim o I Seminário Internacional sobre a Pluridisciplinaridade e a Interdisciplinaridade, que realizou-se na Universidade de Nice/França, em 1970; o Congresso Ciência e Tradição: Perspectivas Transdisciplinares para o século XXI, que ocorreu em Paris no ano de 1991, quando foi cunhado o documento "Ciência e Tradição"; e por fím, o I Congresso Mundial da Transdisciplinaridade, ocorrido em 1994 em Arrábida/Portugal, evento do qual foi extraída a Carta da Transdisciplinaridade (SOMMERMAN, 2005).

O Congresso Internacional de Transdisciplinaridade "Que Universidade para o amanhã? Em busca de uma evolução transdisciplinar da Universidade", ocorrido em 1997, em Locarno/Suíça, produziu um documento síntese, no qual foram sistematizadas definições a respeito da pluridisciplinaridade, da transdisciplinaridade e da interdisciplinaridade em termos epistemológicos. Neste encaminhamento, a interdisciplinaridade foi definida como a transferência de métodos de disciplinas a outras, permitindo maior comunicação entre as áreas cientificas, porém com sua abrangência circunscrita no âmbito disciplinar (SOMMERMAN, 2005).

Do conjunto de encontros e documentos produzidos, ainda restou a interdisciplinaridade, carência de maior definição, sobretudo no que tange a sua aplicação ao conhecimento e cotidiano escolares.

No contexto brasileiro, ressalta-se o papel desempenhado por Hilton Jupiassu na divulgação das reflexões epistemológicas ocorridas em âmbito internacional e a Ivani Fazenda por suas proposições no campo escolar.

Segundo Lenoir (1998), a interdisciplinaridade científica relaciona-se com a produção do conhecimento, enquanto a interdisciplinaridade escolar vincula-se à disseminação do conhecimento. A interdisciplinaridade científica é apresentada por Lenoir (2001) em três perspectivas: a interdisciplinaridade francesa, a americana e a brasileira. Fazenda et al., (2010) refletem sobre essas perspectivas: 


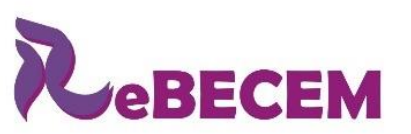

Revista Brasileira de Educação em

Ciências e Educação Matemática

DOI: http://dx.doi.org/10.33238/ReBECEM.2019.v.3.n.3.23547

perspectiva fenomenológica que tem o olhar voltado para a subjetividade dos sujeitos, para a prática e experiência, para a necessidade do autoconhecimento, para o diálogo com o outro, para uma atitude interdisciplinar que alcance uma dimensão humana para o saber-ser (FAZENDA et al.,2010, p. 31).

Hartmann e Zimmermann (2007) discutem a interdisciplinaridade na perspectiva de criação de uma nova inteligência, na capacidade individual dos educadores se abrirem para o diálogo e serem representantes de várias ciências que são articuladas em torno de um único objetivo. Nessa perspectiva, o trabalho com projetos na Educação Básica tornase uma ferramenta adequada para a aproximação de docentes tanto das diversas disciplinas envolvidas na resolução de problemas complexos/cotidianos quanto entre pares; isto pode acarretar na proposição de atividades diversas, permitindo a conexão de saberes específicos e a diminuição das fronteiras entre as "duas culturas", ou seja, entre a educação científica e humanística, proporcionando através do trabalho interdisciplinar, uma nova razão de existência às disciplinas (HARTMANN; ZIMMERMANN, 2007).

A criação de um projeto interdisciplinar impõe desafios, como lidar com a questão de tempo e planejamento, o entusiasmo, a coragem para inovar, a flexibilidade, liderança, formação inicial, formação continuada, projeto político pedagógico (HARTMANN; ZIMMERMANN, 2007), entre outros. Esses pressupostos evidenciam dificuldades reais que afetam a implementação da interdisciplinaridade no contexto escolar.

No entanto, considerando a visão de autores como Lenoir (2001) e Fazenda (2002), o trabalho interdisciplinar no âmbito escolar pode contribuir para a emergência de novos olhares sobre os conhecimentos disciplinares, estabelecendo pontes entre os mesmos e cooperando para execução de projetos que apliquem os conhecimentos específicos de cada disciplina na resolução de questões socioambientais, tão pertinentes aos tempos atuais.

Nesse sentido, Lavaqui e Batista (2007) discutem a compreensão de interdisciplinaridade aplicada à prática educativa. Indicam que há três planos de ação: curricular, didático e pedagógico. O plano curricular refere-se às articulações entre as disciplinas, enquanto a ação didática relaciona-se aos processos de intervenção educativa. $\mathrm{O}$ aspecto pedagógico, por sua vez, trata da aplicação prática dos modelos interdisciplinares no contexto da sala de aula (LAVAQUI; BATISTA, 2007).

Tal ação interdisciplinar pode promover a articulação entre as disciplinas ou áreas de conhecimento e promover vínculos que proporcionem o desenvolvimento de ações mais complexas (SANTOMÉ, 1998). 
DOI: http://dx.doi.org/10.33238/ReBECEM.2019.v.3.n.3.23547

$\mathrm{Na}$ concepção de Fourez (1994) o trabalho interdisciplinar permite uma abordagem mais abrangente das situações cotidianas que, enquanto situações problemas, podem se constituir em ricos pontos de partida para o ensino e a aprendizagem. Nesta vertente, o autor propõe o método de Ilhas Interdisciplinares de Racionalidade, as quais estão articuladas ao domínio das tecnologias e à promoção de habilidades como a comunicação e a autonomia (NEHRING et al., 2002). Tais ilhas podem mobilizar conhecimentos das diversas disciplinas e também os saberes da vida cotidiana para a resolução de uma situação problema adotando, portanto, a abordagem de ensino por projetos e considerando fatores como o contexto em que a escola está inserida.

Diante dessas perspectivas de interdisciplinaridade, o objetivo desta pesquisa é levantar e analisar as argumentações de oito professores da rede pública do Estado de São Paulo, atuantes na área de Ciências da Natureza e Matemática, a respeito do tema "interdisciplinaridade". Procuramos responder as seguintes questões: os discursos de professores atuantes na rede pública têm relação com os conceitos de interdisciplinaridade apresentados por alguns pesquisadores, elencados neste trabalho? De que forma o desenvolvimento de práticas interdisciplinares é entendido pelos professores? Quais justificativas os professores apresentam para a implementação e/ou não implementação de práticas interdisciplinares? Quais conclusões os professores expressam acerca da inserção do processo interdisciplinar na escola?

A seguir, apresentamos a metodologia utilizada nesta pesquisa.

\section{Procedimentos Metodológicos}

Para atender aos objetivos propostos escolhemos realizar uma pesquisa qualitativa, do tipo estudo de caso, em que as pessoas são estudadas em seu ambiente natural (YIN, 2005).

Com o intuito de explorar as diferentes percepções de professores acerca do contexto interdisciplinar na sala de aula, convidadamos oito professores da área de Ciências da Natureza e Matemática de uma escola pública do interior do Estado de São Paulo, os quais são licenciados em Ciências Biológicas, Matemática, Física, Química e Biologia.

Esses docentes são considerados dispostos a desenvolver metodologias diversificadas de ensino e, nas reuniões de professores, costumam participar de discussões acerca da inserção de temas interdisciplinares nas aulas de Ciências da 
DOI: http://dx.doi.org/10.33238/ReBECEM.2019.v.3.n.3.23547

Natureza e Matemática. Dessa forma, interessa-nos levantar suas concepções e verificar quais são seus argumentos acerca da temática dessa pesquisa.

Os dados foram coletados por meio de um questionário aberto em que os professores puderam expor livremente suas argumentações acerca do tema "Interdisciplinaridade", de acordo com suas experiências em sala de aula.

Os dados levantados foram analisados de maneira qualitativa, tendo como referência o padrão de argumentos elaborado por Toulmin:

Figura 1: Elementos do argumento

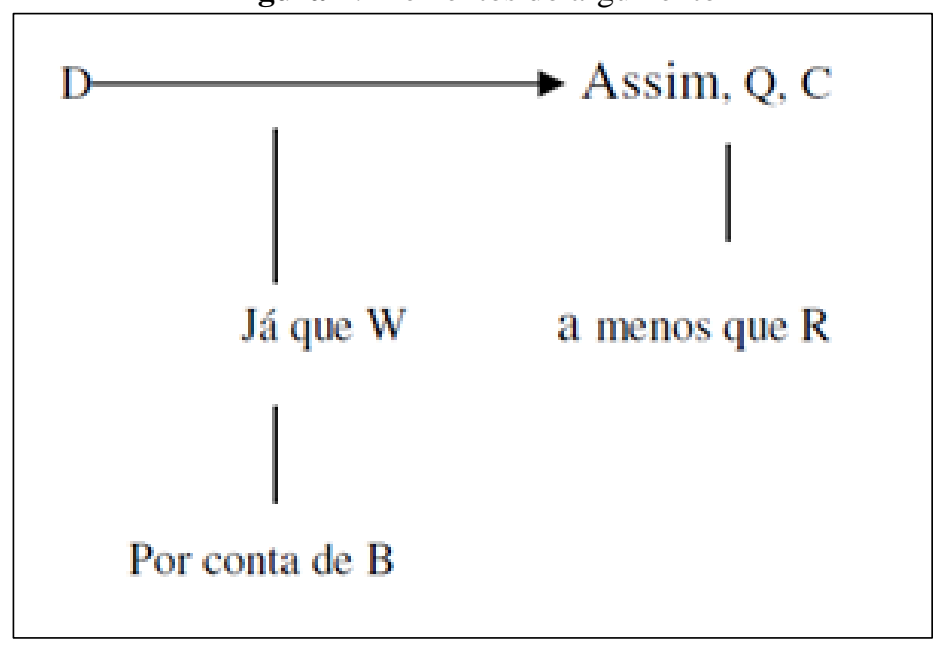

Fonte: Toulmin (2006, p.150)

Na figura 1 estão representados os elementos constitutivos do padrão apresentado por Toulmin (2006), para a análise de argumentos. Um enunciado é considerado como argumento válido se for composto, pelo menos, por três elementos: dado (D), garantia (W) e conclusão (C). Os dados são elementos que propiciam a formação de uma (conclusão). A garantia (W) é uma situação que permite a passagem dos dados à conclusão. Outros elementos podem enriquecer o argumento: o conhecimento (B) que oferece validade à garantia apresentada, por meio de afirmações categóricas; o qualificador modal (Q) que dá força à passagem dos dados à conclusão; e o elemento de refutação (R), o qual traz condições de exceção para a conclusão formada.

Dessa forma, poderemos investigar se conclusões formadas pelos professores, acerca do tema interdisciplinaridade na Educação Básica estão embasadas em justificativas (garantias) relacionadas aos discursos apresentados pelos pesquisadores da área, indicados nesta pesquisa. 
DOI: http://dx.doi.org/10.33238/ReBECEM.2019.v.3.n.3.23547

\section{Resultados e Discussões}

Os professores responderam um questionário, composto por três questões abertas. A seguir, estão representadas as questões e as respectivas respostas de cada docente, os quais foram indicados pela letra $\mathrm{P}$ seguida de um número. Nas falas dos professores fizemos as indicações dos elementos característicos do padrão de Toulmin (2006), os quais estão entre parênteses.

Pergunta 1: Em sua opinião, quais fatores são importantes para o trabalho interdisciplinar em sala de aula? Justifique.

P1: A interdisciplinaridade facilita o trabalho em sala de aula (C) quando há envolvimento por parte de todos os docentes $(\boldsymbol{D})$, quando há sintonia em determinado assunto $(\boldsymbol{W})$, facilita aprendizagem do aluno $(\boldsymbol{C})$.

P2: A possibilidade de trabalhar um tema de diversas formas $(\boldsymbol{D})$, cada disciplina utilizando seus recursos, sua forma de leitura para explorar um projeto comum $(\boldsymbol{W})$. Maior envolvimento dos alunos $(\boldsymbol{C})$, explorando as inteligências múltiplas $(\boldsymbol{Q})$.

P3: Desenvolver no aluno a prática de trabalho em grupo (D) ensinando-os a respeitar a opinião de todos. Através da prática da interdisciplinaridade os conhecimentos ficam mais amplos $(\boldsymbol{C})$, é possível realizar conexões entre as várias disciplinas tornando o aprendizado dos alunos mais interessante $(\boldsymbol{W})$.

P4: $O$ trabalho interdisciplinar auxilia o melhor desempenho do aluno (D) nos conteúdos, amplia a visão sobre os temas (W), contribuindo para uma aprendizagem significativa $(\boldsymbol{C})$.

P5: Professor pode variar suas técnicas de ensinar $(\boldsymbol{D})$, buscando qualidade e não se deter na quantidade de conteúdos $(\boldsymbol{C})$. Professor ser amigo dos alunos $(\boldsymbol{W})$; valorizar as questões sociais como: dignidade, caráter, bondade e honestidade (W); próprio aluno ser capaz de elaborar os próprios conteúdos de aprendizagem $(\boldsymbol{W})$; aluno vai ser sempre um agente da aprendizagem $(\boldsymbol{W})$, sempre se atualizando, pesquisando, aprofundando e melhorando seu conhecimento $(\mathbf{Q})$.

P6: Os conteúdos $(\boldsymbol{D})$ que podem se relacionar $(\boldsymbol{C})$, principalmente Química e Física.

P7: A abordagem, a contextualização $(\boldsymbol{D})$, processos de ensino e aprendizagem mais interessante $(\boldsymbol{C})$, pois sempre resgata o que já foi construído e acrescentando novas informações, conhecimentos $(\boldsymbol{W})$.

P8: A integração das disciplinas e a contextualização no ensino e aprendizagem (D). A aula ficar mais interessante $(\boldsymbol{C})$, pois a aprendizagem pode ser mais significativa para os alunos $(\boldsymbol{W})$, ficando mais fácil de trabalhar com o cotidiano $(\mathbf{Q})$. Por exemplo, inserir na matemática temas sociais e atuais.

Análise das argumentações:

Diante do tema interdisciplinaridade, os professores P2, P3, P5, P6, P7 e P8 apresentaram dados (D) relacionados à metodologia de ensinar, como trabalho diversificado, trabalho em grupo, relação entre conteúdos e contextualização. Todos apresentaram garantias (W) otimistas, como exploração de um objeto, tornar o aprendizado mais interessante, aluno ser agente da aprendizagem, aprendizagem significativa. As conclusões (C) também são positivas, pois os professores relacionaram 
DOI: http://dx.doi.org/10.33238/ReBECEM.2019.v.3.n.3.23547

o assunto interdisciplinaridade com o maior envolvimento dos alunos, ampliação dos conhecimentos, qualidade e relação entre conteúdos e aprendizagem mais interessante.

$\mathrm{O}$ argumento do professor P1 traz como dado (D) o envolvimento dos docentes por meio das práticas interdisciplinares. Aponta como garantia (W) a sintonia dos docentes em determinados assuntos. A conclusão (C) do professor está relacionada à aprendizagem do aluno. O professor P4 indica como dado importante do trabalho interdisciplinar, o desempenho do aluno. A conclusão (C) está relacionada à aprendizagem significativa, devido ampliação de visão sobre os temas.

Entendemos que a maioria desses argumentos está relacionada com os planos de ação, plano curricular e didático indicados por Lavaqui e Batista (2007) e que os conceitos apontados como importantes para uma prática interdisciplinar se aproximam da dimensão brasileira, em que é importante o diálogo e há valorização da dimensão humana para o saber-ser (FAZENDA et al., 2010).

A seguir, apresentamos a segunda questão feita aos professores, as suas respostas e respectivas análises.

Pergunta 2: Em sua opinião, quais fatores prejudicam o trabalho interdisciplinar em sala de aula?

P1: A falta de envolvimento dos membros (D).

P2: A dificuldade dos professores se reunirem para organizar atividades que atendam todas as disciplinas envolvidas (D). Há um começo, mas no decorrer do trabalho o tempo e as condições do professor (trabalha em várias escolas) (W) dificultam o desenvolvimento do projeto $(\mathbf{C})$.

P3: A falta de tempo entre os professores das áreas para realizar encontros (D) que seriam necessários para a elaboração dos projetos e troca de experiências (C).

P4: O principal fator [que prejudica o trabalho interdisciplinar] é a falta de sintonia entre os conteúdos das disciplinas no currículo $(\mathbf{C})$.

P5: Falta de diálogo entre os próprios colegas (D); indisciplina por parte de alguns alunos (D);

P6: A falta de integração entre as disciplinas (D).

P7: O tempo para preparar, os materiais que não temos como impressora, papel sulfite, entre outros $(\boldsymbol{D})$. Por isso, muitas vezes há uma resistência por parte dos professores em relação a esse assunto $(\mathbf{C})$.

P8: Os próprios professores não abrem esse leque (D), talvez por resistência $(\boldsymbol{C})$, pela falta de incentivo pela direção (W) ou porque dão [as atividades interdisciplinares] mais trabalho preparar as aulas $(\mathbf{W})$.

Análise das argumentações:

As respostas dos professores P1, P8 e P5 indicam dados (D) que revelam a falta de envolvimento dos docentes em projetos interdisciplinares e também que há a falta de diálogo entre os próprios professores. O P8 conseguiu expressar um argumento, pois a partir do dado (D) houve a formação de uma conclusão (C): os professores não se 
DOI: http://dx.doi.org/10.33238/ReBECEM.2019.v.3.n.3.23547

envolvem nos projetos interdisciplinares por "resistência". O docente apontou também as possíveis garantias (W) para tal conclusão: "falta de incentivo pela direção ou porque dão [as atividades interdisciplinares] mais trabalho preparar as aulas".

Os professores P2, P3 e P7 indicam dados referentes às dificuldades de implementação de atividades interdisciplinares na escola, os quais estão relacionados com questões de falta de tempo para que aconteça reuniões entre os docentes. O professor P3 conclui (C) que a questão do tempo é importante para haver trocas de experiências entre eles. O professor P7 conclui (C) que muitas vezes há uma resistência por parte dos professores em relação a esse assunto. Essa conclusão foi formada a partir do dado relacionado à falta de tempo e também de materiais e recursos.

Os professores P4 e P6 revelam que o trabalho interdisciplinar é prejudicado devido a dados (D) referentes à falta de sintonia entre as disciplinas do currículo.

Diante do exposto, vemos que os professores apontam dificuldades para a execução de projetos interdisciplinares, pois possuem uma visão de que a interdisciplinaridade está articulada com a troca de experiências por meio do diálogo com o outro, numa perspectiva de interdisciplinaridade na concepção brasileira. Dessa forma, os profissionais reconhecem a interdiciplinaridade como uma estratégia de ensino que necessita de envolvimento ativo dos participantes.

No entanto, considerando o contexto de que os professores são vistos na escola como profissionais dispostos à mudança, não há indicação de métodos que possam contribuir para a superação de tais obstáculos.

Pergunta 3: Por fim, defina o que você entende por interdisciplinaridade e defenda a sua posição sobre as práticas interdisciplinares.

P1: É uma maneira inteligente no processo de ensino e aprendizagem desenvolvido no ambiente escolar $(\mathbf{C})$, onde as diversas disciplinas se interagem buscando o bem comum aos alunos $(\mathbf{W})$.

P2: Interdisciplinaridade é a forma de relacionar conteúdos de diversas disciplinas tendo um tema, foco comum norteador das ações (C). É uma possibilidade de perceber como uma disciplina pode usar seus recursos para auxiliar as demais, ampliando a aprendizagem (C). Não são isoladas, mas sim planejadas, elaboradas conjuntamente para garantir uma aprendizagem significativa (W). O que não é tarefa fácil em nosso cotidiano escolar. Infelizmente vemos cada professor trabalhando isoladamente devido a falta de tempo, acúmulo de escolas, ou mesmo dificuldade de trabalhar coletivamente com projetos $(\mathbf{R})$.

P3: Interdisciplinaridade em minha opinião seria construir o conhecimento de uma forma ampla $(\mathbf{C})$, unindo colaborações de todas as disciplinas das diferentes áreas do conhecimento, para alcançar um mesmo objetivo (W). Sem dúvidas com a interdisciplinaridade quem ganha são os alunos $(\mathbf{C})$, pois aprenderiam de uma forma mais prazerosa e prática $(\mathbf{W})$. Mas infelizmente está distante de acontecer devido a sistematização do sistema $(\mathbf{R})$. 
DOI: http://dx.doi.org/10.33238/ReBECEM.2019.v.3.n.3.23547

P4: Trabalho interdisciplinar é aplicar em várias disciplinas um tema que possa ser trabalhado nos mesmos, dentro de cada particularidade e abordagem curricular $\mathbf{( C )}$.

P5: A interdisciplinaridade ocorre por meio da integração, diálogos, proximidade convergência, contato e interação $(\boldsymbol{D})$. Ela proporciona uma aprendizagem muito mais estruturada e rica $(\mathbf{C})$, pois os conceitos estão organizados em torno de unidades mais globais de estruturas conceituais e metodológicas compartilhadas por várias disciplinas $(\mathbf{W})$. As práticas interdisciplinares em sala podem ser muito válidas, pois proporcionam aos alunos a capacidade de construir os próprios conceitos $(\mathbf{Q})$.

P6: A relação entre os conteúdos das diversas disciplinas $(C)$, mas para haver essa relação deveria acontecer mais diálogos entre os programas, o que na prática não se verifica $\mathbf{( R )}$.

P7: É o processo de ligação entre as disciplinas. Essa ligação é muito importante (C) porque sempre vem para acrescentar, para ajudar e melhorar o conhecimento dos envolvidos $(\mathbf{W})$.

P8: É o envolvimento de todas as disciplinas em uma escola, e não isoladamente como a maioria faz $(\boldsymbol{D})$. Acho que todos os professores deveriam pensar e adotar essa prática que é muito favorável no nosso trabalho $(\mathbf{C})$, pois podemos inserir conteúdos de outras disciplinas nas aulas de matemática, como geografia, história, física, ciências, artes e outras (W), o que pode oferecer aos alunos uma compreensão mais ampla da realidade $(\mathbf{Q})$.

Análise das argumentações:

As respostas dadas pelos professores evidenciam a construção de argumentos válidos de acordo com o padrão de Toulmin (2006). Os professores P2, P3 e P6 expressaram argumentos com a presença de elementos de refutação $(\mathrm{R})$, os quais dão uma qualidade maior para os argumentos formados. O professor P2 ressalta em suas conclusões $(C)$ o papel norteador que as atividades interdisciplinares possuem e a função de ampliar a aprendizagem. No entanto, indica que a falta de integração entre os docentes prejudica as ações interdisciplinares $(\mathrm{R})$. O professor P3 apresenta conclusões $(\mathrm{C})$ que também estão relacionadas ao aspecto de construção de conhecimento pelos alunos, por meio das atividades interdisciplinares. O professor refuta $(\mathrm{R})$ sua conclusão, indicando a sistematização do sistema. Tal refutação também é apresentada pelo professor P6, pois indica que os programas de ensino não possuem diálogos.

Os professores P4 e P7 concluem (C) em suas respostas que o uso de atividades interdisciplinares está relacionado à articulação entre as disciplinas. O Professor P7 justifica (W) que tais atividades podem melhorar os conhecimentos das pessoas envolvidas.

Os argumentos formados pelos professores mostram que há uma valorização das práticas interdisciplinares no contexto educativo. No entanto, tais ações não são desenvolvidas de maneira real na escola, devido a diversos fatores que impedem seu desenvolvimento. 


\section{Considerações finais}

O estudo realizado traz evidências de que o trabalho com foco interdisciplinar é visto por alguns professores como uma maneira de propiciar o diálogo, a interatividade entre os diversos saberes, em busca da autonomia, como aponta Nehring et al., (2002). No entanto, a argumentação expressa pelos docentes revela que a prática de ações interdisciplinares está comprometida por fatores, como a sistematização do sistema, pela falta de integração entre os membros que atuam na escola, entre outras causas.

Embora existam muitas dificuldades na implementação de atividades interdisciplinares na escola, parece-nos que há a expressão de argumentos que revelam acreditar no potencial transformador que as atividades interdisciplinares possuem, fazendo emergir a dimensão humana do saber-ser, conforme os estudos de Fazenda (2002). Dessa forma, os profissionais indicam que o trabalho com atividades interdisciplinares é importante para o desenvolvimento do aluno, no entanto, os fatores dificultadores são vistos como uma barreira para que acontece a emergência de atividades de cunho interdisciplinar na área de Ciências da Natureza e Matemática.

Dessa forma, acreditamos que esse estudo pode trazer contribuições para as discussões acerca da temática relacionada à interdiciplinaridade nos espaços da sala de aula da Educação Básica. Possibilita também a reflexão acerca de novos estudos que priorizem a formação de professores para saberem lidar com as adversidades cotidianas e colocar em prática os princípios da interdisciplinaridade, na perspectiva brasileira, com o viés do autoconhecimento e diálogo com o outro (FAZENDA et al.,2010).

\section{Referências}

FAZENDA, I. C. A. (org.). Dicionário em construção: Interdisciplinaridade. 2. ed. SP: Cortez, 2002.

FAZENDA, I. C. et al. Avaliação e interdisciplinaridade. Revista Interdisciplinaridade, São Paulo, v.1, n. 0, p.01-83, 2010.

FOUREZ, G. Alfabétisation scientifique et tecnique. Essai sur les finalités de l'enseignement des sciences. Belgique: De Boeck Université, 1994.

HARTMANN, A. M.; ZIMMERMANN, E. O trabalho interdisciplinar no Ensino Médio: a reaproximação das "duas culturas". Revista Brasileira de Pesquisa em Educação em Ciências, Belo Horizonte, v. 7, n. 2, s.p., 2007.

LAVAQUI, V; BATISTA, I. L. Interdisciplinaridade em ensino de ciências e de matemática no ensino médio. Ciência \& Educação, Bauru, v. 13, n. 3, p. 399-420, 2007. 
LENOIR, Y. Didática e Interdisciplinaridade: uma complementaridade necessária e incontornável. In: FAZENDA, I. (org.). Didática e interdisciplinaridade. São Paulo: Papirus, 1998.

LENOIR, Y. Lês Fondaments de l'interdisciplinaritè dans la formation à l'enseignement. Universite de Sherbrooke: Editions Du CRP, 2001.

NEHRING, C. M. et al. As ilhas de racionalidade e o saber significativo: o ensino de ciências através de projetos. ENSAIO - Pesquisa em Educação em Ciências, Belo Horizonte, v. 2, n. 1, p. 88-105, 2002.

SANTOMÉ, J. T. Globalização e interdisciplinaridade: o currículo integrado. Porto Alegre: Artes Médicas, 1998.

SNOW, C. P. As duas culturas e uma segunda leitura: uma visão ampliada das duas culturas e a revolução científica. São Paulo: EDUSP, 1995.

SOMMERMAN, A. A inter e a transdisciplinaridade. In: SEMINÁRIO INTERNACIONAL DE EDUCAÇÃO "INTERDISCIPLINARIDADE COMO FORMA DE INCLUSÃO NUMA EDUCAÇÃO MUNDIAL”, 10., 2005, Cachoeira do Sul. Anais.. Cachoeira do Sul, 2005.

TOULMIN, S. E. Os usos do argumento. 2. ed. São Paulo: Martins Fontes, 2006.

YIN, R. K. Estudo de caso: planejamento e métodos. 3. ed. São Paulo: Martins Fontes, 2005.

Recebido em: 10 de novembro de 2019.

Aceito em: 26 de novembro de 2019. 